\title{
Allicin activates autophagic cell death to alleviate the malignant development of thyroid cancer
}

\author{
YANGFENG XIANG, JIANQIANG ZHAO, MING ZHAO and KEJING WANG \\ Department of Head and Neck Surgery, Zhejiang Cancer Hospital, Hangzhou, Zhejiang 310022, P.R. China
}

Received January 16, 2017; Accepted September 13, 2017

DOI: $10.3892 /$ etm.2018.5828

\begin{abstract}
Allicin has been reported to inhibit cancer cell proliferation, induce cell apoptosis and enhance the accumulation of reactive oxygen species. However, it has remained elusive whether allicin improves multidrug resistance in thyroid cancer cells through modulating autophagy. The present study demonstrated that combined use of allicin and cisplatin or carboplatin resulted in an enhanced growth inhibitory effect on SW1736 and HTh-7 cells. Furthermore, treatment with allicin significantly increased SW1736 and HTh-7 cell autophagy. Of note, allicin-induced cell death was largely abolished by 3-methyladenine or chloroquine treatment, suggesting that allicin-induced A549 cell death was dependent on autophagy. Western blot analysis demonstrated that allicin treatment inhibited the activation of Akt, mammalian target of rapamycin and S6. Furthermore, it was demonstrated that combined use of allicin and rapamycin induced more cell death compared with that induced by allicin or rapamycin alone. In conclusion, allicin may serve as an adjunctive therapy for thyroid cancer, as it induces autophagy-dependent cell death even when cancer cells have developed apoptosis resistance.
\end{abstract}

\section{Introduction}

Follicular cell-derived thyroid cancer is ranked as the most prevalent endocrine malignancy (1). The majority of deaths associated with thyroid cancer occur in patients with poorly differentiated and other aggressive forms of papillary thyroid carcinoma (PTC). In addition, rare anaplastic thyroid cancer (ATC) demonstrates a poor survival rate with mortality usually occurring within 6 months of diagnosis (2). Loss of uptake and trapping ability of radioactive iodine has been suggested to be a hallmark of these cancers, rendering them resistant to the best systemic therapy available for thyroid cancer. Furthermore, thyroid cancer frequently exhibits resistance to

Correspondence to: Dr Kejing Wang, Department of Head and Neck Surgery, Zhejiang Cancer Hospital, 38 Guangji Road, Hangzhou, Zhejiang 310022, P.R. China

E-mail:m13375483602@163.com

Key words: allicin, autophagy, multidrug resistance, cell death standard chemotherapy regiments (3). Therefore, it is of great importance to identify novel therapeutic methods to address and overcome the drug resistance of thyroid cancer.

In the progression of tumors, autophagy is regarded as a double-edged sword. At the initial development phase, autophagy functions as a tumor suppressor mechanism $(4,5)$. However, it prompts the malignant progression of tumors in hypoxic microenvironments $(6,7)$. When autophagy is initiated, cytosolic light chain (LC)3 (LC3-I) loses a small polypeptide during enzymatic hydrolysis and is transformed into the membrane type of LC3, LC3-II, thereby enhancing the formation of an autophagosome. Thus, the LC3I/LC3II ratio may be utilized to estimate the level of autophagy (8). Clinical studies have demonstrated that autophagy is enhanced after cancer therapy, and is therefore involved in the development of chemotherapy resistance $(9,10)$. The modulation of the extent of autophagy may therefore provide a novel approach for the treatment of tumor cells.

Allicin is isolated from garlic and may also be extracted as a brownish orange dry resin from the Garcinia hanburyi tree occurring in South-East Asia $(11,12)$. In previous years, Asians have used allicin as a traditional medicine for the therapy of various diseases. Allicin has been reported to possess anti-infectious, anti-oxidant, anti-inflammatory and anti-viral effects (13). Allicin has also been evaluated as an anti-tumor agent in vitro against malignancies including lung cancer, leukemia, colorectal cancer, prostate cancer and hepatocellular carcinoma $(14,15)$. It has been reported that allicin inhibited cancer cell proliferation, induced cell apoptosis and enhanced the accumulation of reactive oxygen species (16-18). However, whether allicin improves multidrug resistance of thyroid cancer through modulating autophagy has remained elusive.

The present study explored the effects of combined use of allicin and cisplatin (CDPP) or carboplatin (CBP) on thyroid cancer cells. The results indicated that allicin activated autophagy and suppressed the viability of thyroid cancer cells, suggesting an anti-tumor effect of allicin in thyroid cancer cells through modulating autophagy.

\section{Materials and methods}

Cell lines and culture. SW1736 (BRAFV600 ${ }^{\mathrm{E} / \mathrm{wt}}$ ) and HTh-7 (NRAS ${ }^{\mathrm{Q} 61 \mathrm{R}}$ ) cells (both American Type Culture Collection, Manassas, VA, USA) were cultured in Dulbecco's modified 
Eagle's medium (DMEM)/F-12 (Hyclone, Logan, UT, USA) supplemented with $10 \%$ heat-inactivated fetal calf serum (Gibco; Thermo Fisher Scientific, Inc., Waltham, MA, USA), $100 \mathrm{U} / \mathrm{ml}$ penicillin and streptomycin in $25-\mathrm{cm}^{2}$ culture flasks at $37^{\circ} \mathrm{C}$ in a humidified atmosphere with $5 \% \mathrm{CO}_{2}$. Prior to treatment, the chemotherapeutic agent CDDP (cat. no. 15663-27-1; Sigma-Aldrich; Merck KGaA, Darmstadt, Germany) or CBP (cat. no. 41575-94-4; Sigma-Aldrich; Merck KGaA) was dissolved in DMEM (Gibco; Thermo Fisher Scientific, Inc.) to prepare a stock solution $(1 \mathrm{mM})$, which was added directly to the media at the indicated concentrations.

MTT colorimetric assay. To investigate the influence of allicin on cancer cell chemosensitivity, SW1736 and HTh-7 cells were seeded in 96-well tissue culture plates at a density of $5 \times 10^{4}$ cells per well in DMEM medium. When the confluence reached $70 \%$ confluence, $10 \mu \mathrm{M}$ CDPP or $10 \mu \mathrm{M}$ CBP or $10 \mu \mathrm{M}$ allicin (cat. no. 539-86-6; Yuanmu (Shanghai, China), was added to each well and the cells were incubated at $37^{\circ} \mathrm{C}$ for $48 \mathrm{~h}$.

To explore whether enhanced SW1736 and HTh-7 cell death was partially attributed to allicin $(10 \mu \mathrm{M})$-induced cell autophagy, 3-methyladenine (3-MA; $5 \mathrm{mM}$ ) or chloroquine (CQ; $40 \mu \mathrm{M})$ an autophagolysosome fusion inhibitor, were administered to SW1736 and HTh-7 cells for $24 \mathrm{~h}$ at $37^{\circ} \mathrm{C}$.

Cell viability was examined with MTT assay kits (Sigma-Aldrich; Merck KGaA). The blue formazan products in the cells were dissolved in dimethylsulfoxide and spectrophotometrically measured at a wavelength of $550 \mathrm{~nm}$. All experiments were performed in triplicate.

Transient transfection with green fluorescence protein (GFP)-LC3. SW1736 and HTh-7 cells were seeded in 6-well plates at a density of $5 \times 10^{5}$ cells/well, followed by incubation for $24 \mathrm{~h}$. Subsequently, a GFP-LC3II expression plasmid (cat. no. 17-10230; Merck KGaA, Darmstadt, Germany) was transfected into cells using Lipofectamine $2000^{\circledR}$ reagent (Invitrogen, Carlsbad, CA, USA) according to the manufacturer's protocol. To explore whether allicin exerts a synergistic effect on the cytotoxity of CDDP or CBP cells were simultaneously treated with CDDP or CBP in the presence or absence of allicin for $48 \mathrm{~h}$. In preliminary studies performed by the authors, no differences were identified between pretreatment with allicin followed by platinum drug and simultaneous treatment with allicin and the platinum drugs when Ad-GFP-LC3II plasmid was transfected into SW1736 and HTh-7 cells (data not shown). Therefore, in the present study simultaneous treatment with allicin and the platinum drugs was performed. In brief, following $24 \mathrm{~h}$ the cells were treated with $10 \mu \mathrm{M}$ allicin in the presence or absence of cisplatin $(10 \mu \mathrm{M})$ or $10 \mu \mathrm{M}$ carboplatin for $48 \mathrm{~h}$ at $37^{\circ} \mathrm{C}$. Subsequently, GFP-LC3II-positive autophagosomes were counted under a confocal laser microscope (LSM700; Carl Zeiss, Jena, Germany). Cells without any treatment were used as the control, however they were also transfected with the GFP-LC3II plasmid as described above.

Electron microscopy. Following $10 \mu \mathrm{M}$ allicin treatment in the presence or absence of $5 \mathrm{mM} 3$-MA for $48 \mathrm{~h} \mathrm{SW1736}$ and HTh-7 cells were fixed with $2 \%$ glutaraldehydeparaformaldehyde in $0.1 \mathrm{M}$ PBS (pH 7.4) for $12 \mathrm{~h}$ at $4^{\circ} \mathrm{C}$ and then washed three times for $30 \mathrm{~min}$ in $0.1 \mathrm{M}$ PBS. Subsequently, the samples were postfixed with $1 \% \mathrm{OsO}_{4}$ dissolved in $0.1 \mathrm{M}$ PBS for $2 \mathrm{~h}$ and dehydrated in an ascending gradual series of ethanol (50-100\%) and soaked with propylene oxide. Specimens were embedded using a Poly/Bed 812 kit (Polysciences, Warrington, PA, USA). After pure fresh resin embedding and polymerization at $60^{\circ} \mathrm{C}$ in an electron microscope oven (TD-700; Dosaka, Kyoto, Japan) for 24 h, 350-nm sections were cut with a Leica Ultracut UCT Ultra-microtome (Leica Microsystems, Wetzlar, Germany), stained with toluidine blue for light microscopy. Furthermore, $70-\mathrm{nm}$ thin sections were double-stained with $7 \%$ uranyl acetate and lead citrate for contrast staining. All thin sections were observed under a transmission electron microscope (JEM-1011; JEOL, Tokyo, Japan) at an acceleration voltage of $80 \mathrm{kV}$.

Western blot analysis. SW1736 and HTh-7 cells were treated with $10 \mu \mathrm{M}$ allicin in the presence or absence with $10 \mu \mathrm{M}$ rapamycin for $48 \mathrm{~h}$ at $37^{\circ} \mathrm{C}$. Total protein samples were extracted from the cultured cells using an radioimmunoprecipitation assay buffer (Beijing Solarbio Science \& Technology Co., Ltd., Beijing, China) according to the manufacturer's protocol. Immunoblotting was performed as previously described (8). The primary antibodies, including anti-p62 (cat. no. 39749; 1:1,000; Cell Signaling Technology, Inc., Danvers, MA, USA), anti-LC3I and II (cat. no. L8918, $1: 1,000$ ) and anti- $\beta$-actin (cat. no. A5441; $1: 3,000$ ) (both Sigma-Aldrich; Merck KGaA). The secondary antibodies were horseradish peroxidase-conjugated goat anti-rabbit immunoglobulin G (ZF-0311; 1:5,000; OriGene Technologies, Inc. Beijing, China).

Statistical analysis. Values are expressed as the mean \pm standard error. Multiple group comparisons were made by one-way analysis of variance followed by Tukey's post-hoc test using GraphPad version 6.0 software (GraphPad Software, Inc., La Jolla, CA, USA). $\mathrm{P}<0.05$ was considered to indicate a statistically significant difference.

\section{Results}

Allicin inhibits thyroid cancer cell growth in a synergistic manner with CDDP. First, the growth inhibitory effect of CDPP on SW1736 and HTh-7 cells was assessed using an MTT assay after they had been exposed to allicin for $48 \mathrm{~h}$. As presented in Fig. 1A and B, treatment with CDDP, carboplatin (CBP) or allicin alone significantly suppressed SW1736 and HTh-7 cell viability. To explore whether allicin exerts a synergistic effect on the cytotoxity of CDDP or CBP, cells were simultaneously treated with CDDP or CBP in the presence or absence of allicin for $48 \mathrm{~h}$. These results indicated that combination use of allicin and CDDP or CBP resulted in an enhanced growth inhibitory effect on SW1736 and HTh-7 cells (Fig. 1A and B).

Allicin induces autophagy in SW1736 and HTh-7 cells. The present study then explored the cytotoxic effects of allicin on SW1736 and HTh-7 cells. First, the expression of autophagy markers, including LC3II and p62, was explored. Pre-incubation with allicin significantly increased the level of LC3II, while 
A

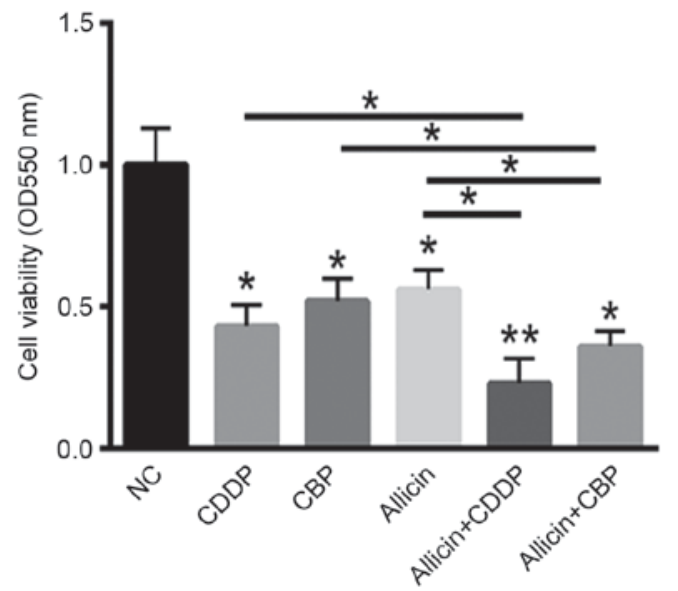

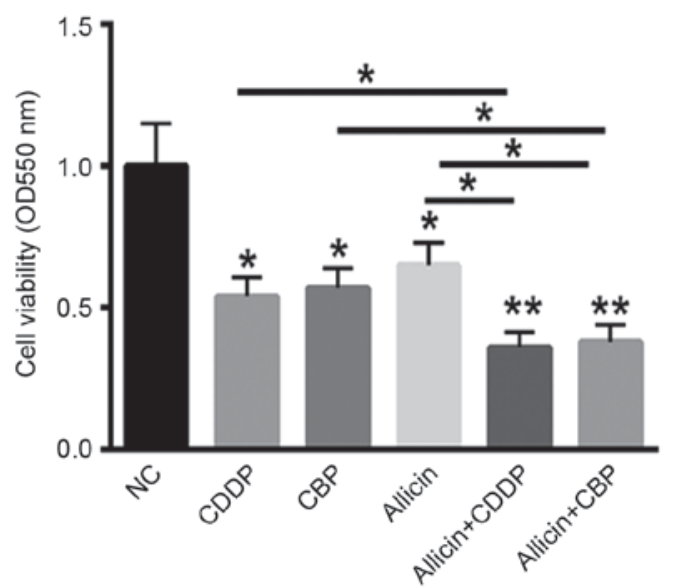

Figure 1. Allicin inhibits thyroid cancer cell growth and exerts a synergistic effect with CDDP or CBP. An MTT assay demonstrated that combined use of allicin and CDDP or CBP resulted in an enhanced growth inhibitory effect on (A) SW1736 and (B) HTh-7 cells. ${ }^{*} \mathrm{P}<0.05$, ${ }^{* *} \mathrm{P}<0.01$ vs. control or as indicated. CDPP, cisplatin; CBP, carboplatin; NC, negative control.

the expression of p62 was significantly suppressed in SW1736 cells compared with the control (Fig. 2A). A significant reduction in $\mathrm{p} 62$ was also observed in the HTh-7 cells (Fig. 2A). In addition, treatment with 3-MA, a specific autophagy inhibitor, markedly abolished allicin-induced SW1736- and HTh-7-cell autophagy (Fig. 2A). Transmission electron microscopy also demonstrated enhanced autophagy vesicles in SW1736 and HTh-7 cells treated with allicin (Fig. 2B). In addition, transfection with GFP-LC3 was employed to detect autophagy. As presented in Fig. 2C, treatment with allicin significantly enhanced the autophagosomes in SW1736 and HTh-7 cells. This effect was suppressed by addition of 3-MA, indicating allicin-induced autophagy through LC3 accumulation.

Allicin-induced SW1736 and HTh-7 cell death is associated with severe autophagy. To explore whether enhanced SW1736 and HTh-7 cell death was partially attributed to allicin (10 $\mu \mathrm{M})$-induced cell autophagy, 3-MA (5 mM) or chloroquine $(\mathrm{CQ}, 40 \mu \mathrm{M})$, an autophagolysosome fusion inhibitor, were applied (Fig. 3). Allicin-induced cell death was largely abolished by 3-MA in a dose-dependent manner in SW1736 and HTh-7 cells Fig. 3A and C. Suppression of autophagy by CQ also largely repressed the allicin-induced death of SW1736 and HTh-7 cells (Fig. 3B and D). These results demonstrated that allicin-induced SW1736 and HTh-7 cell death was dependent on autophagy.

Allicin suppresses Akt/mammalian target of rapamycin (mTOR) signaling in SW1736 and HTh-7 cells. Previous studies have indicated that the Akt/mTOR signaling pathway has a key role in the process of autophagy (19). Thus, the present study evaluated the activation of Akt/mTOR signaling after allicin treatment. Western blot analysis demonstrated that allicin treatment significantly reduced the phosphorylation level of Akt, mTOR and S6, indicating the inactivation of the Akt/mTOR signaling pathway by allicin treatment in SW1736 and HTh-7 cells (Fig. 4A). Subsequently the potential synergistic effect of allicin and rapamycin on SW1736 and HTh-7 cell autophagy was explored. As presented in Fig. 4B, combination treatment with allicin and rapamycin significantly enhanced the LC3II/LC3I ratio in SW1736 and HTh-7 cells compared with that in the groups treated with allicin or rapamycin alone. The synergistic effect of allicin and rapamycin on SW1736 and HTh-7 cell death was also explored. Of note, combined use of allicin and rapamycin induced significantly more cell death compared with that induced by allicin or rapamycin alone (Fig. 4C).

\section{Discussion}

The incidence of thyroid cancer is continuing to increase worldwide (20). Compared with males, females of various ethnicities demonstrate a higher incidence of thyroid cancer $(21,22)$. At present, platinum-based drugs are widely applied for the treatment of thyroid cancer patients in the clinic. Unfortunately, it is common for these patients to develop drug resistance $(23,24)$. Most thyroid cancer patients who develop incurable metastasis are candidates for neoadjuvant or palliative chemotherapy $(20,25)$. However, two thirds of these patients still benefit from conventional chemotherapy. Studies have indicated that conventional chemotherapy did not achieve apoptosis-dependent cell death in these thyroid cancer patients $(26,27)$. In this regard, resistance to platinum-based drugs among these patients mainly arises from deficiency of apoptotic pathways in their thyroid cancer cells $(26,27)$. Thus, it is important to explore novel drugs with the ability to enhance thyroid cell death, such as autophagy inducers.

In addition to apoptosis-dependent cancer cell death, autophagic cell death has been greatly valued as a novel mechanism for cancer cell death induced by chemotherapeutics $(6,7,9)$. Certain studies suggest that the pro-survival functions of autophagy decrease the efficacy of cancer therapeutics $(20,25)$. However, other studies have demonstrated that autophagy also induced cancer cell death after various treatments (9,28-30). Thus, activation of autophagic cell death is becoming an important strategy for cancer treatment. 
A

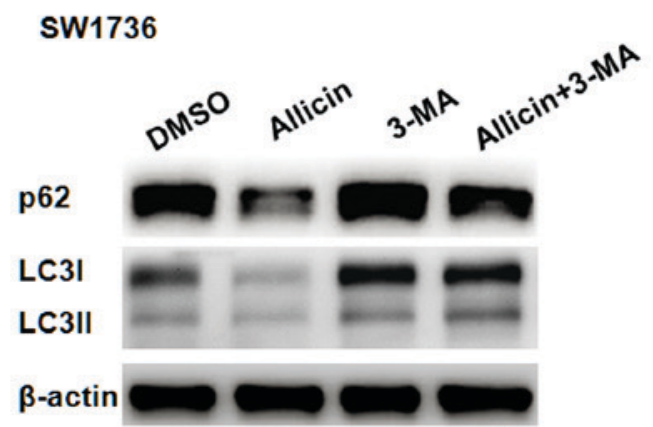

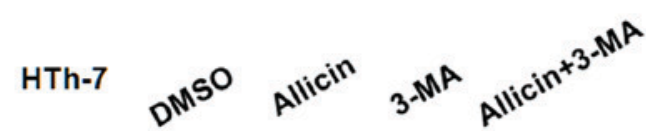

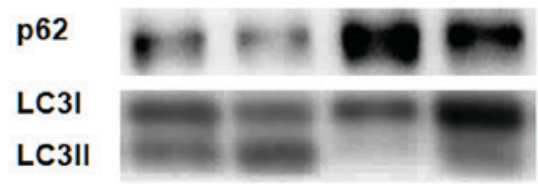

$\beta$-actin

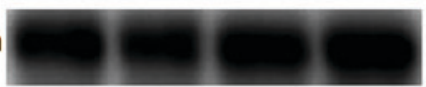

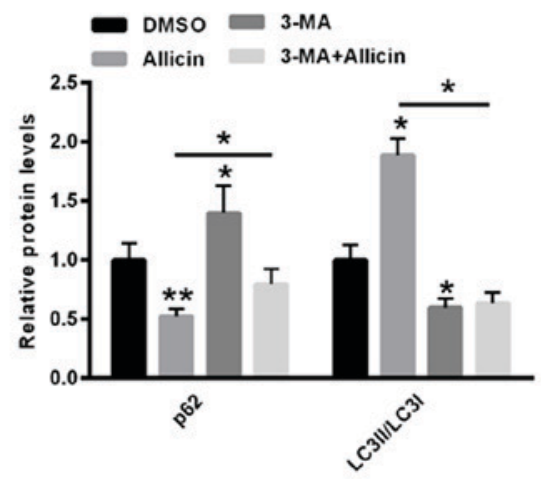

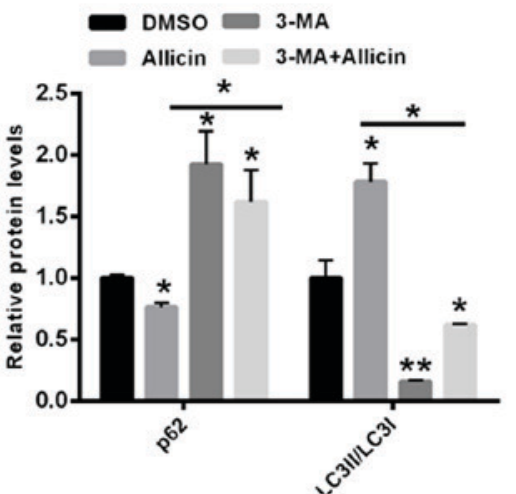

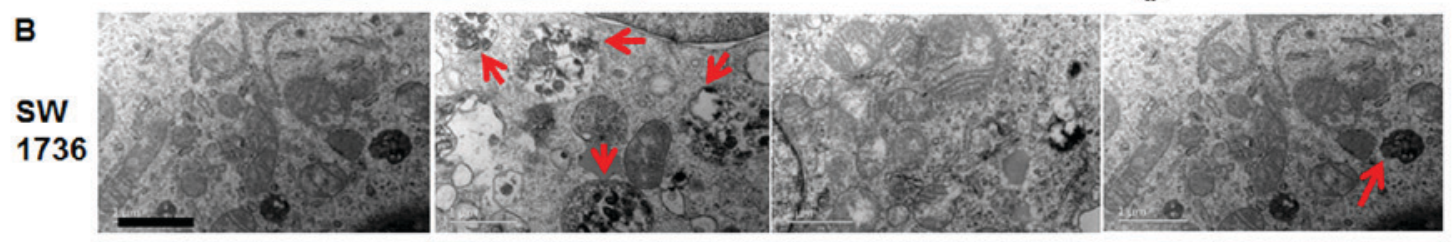

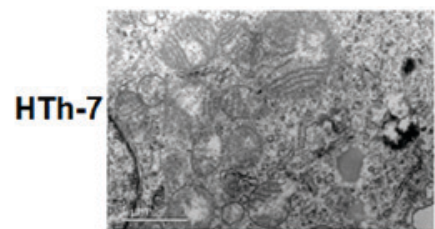

DMSO
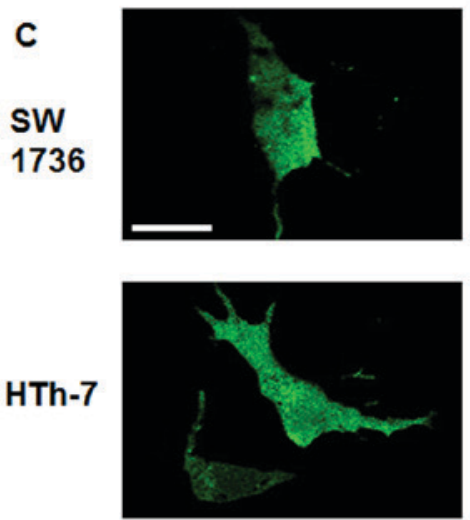

DMSO

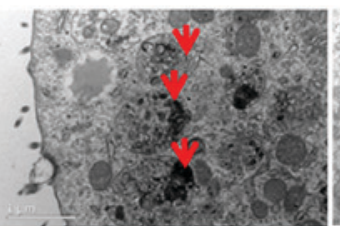

Allicin
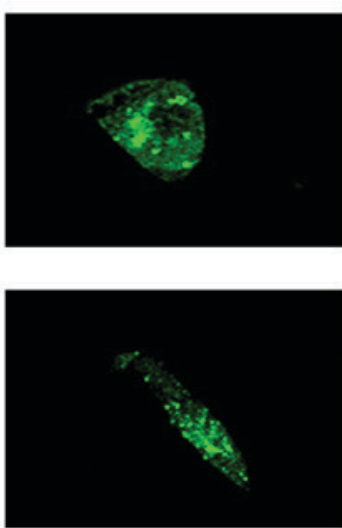

Allicin

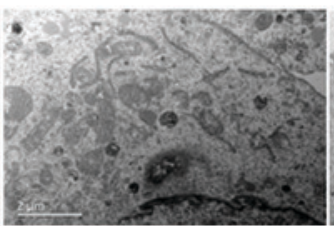

3-MA
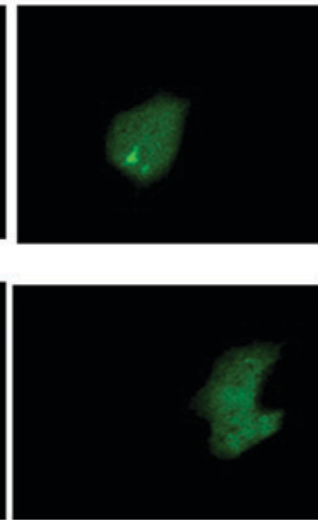

3-MA

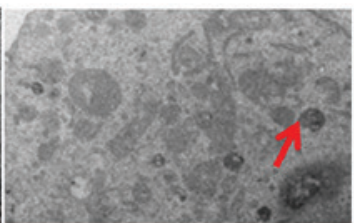

3-MA+allicin
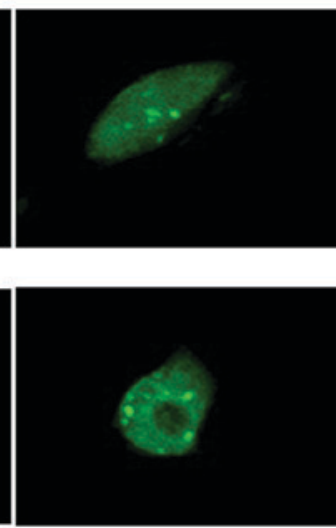

3-MA

Figure 2. Allicin induces autophagy in SW1736 and HTh-7 cells. (A) Western blot analysis. (B) Transmission electron microscopy detection (scale bar, $1 \mu \mathrm{m}$ ). Red arrows indicate autophagsomes. (C) Green fluorescence protein-LC3 transfection (scale bar applies to all images, $10 \mu \mathrm{m}$ ). ${ }^{*} \mathrm{P}<0.05,{ }^{* *} \mathrm{P}<0.01 \mathrm{vs}$. control or as indicated. DMSO, dimethylsulfoxide; 3-MA, 3-methyladenine; LC3, light chain 3.

The present study explored the efficacy of allicin in the treatment of thyroid cancer. Compared with CDDP or CBP, the inhibitory effects of allicin on cell viability were lower.
However, combined use of allicin and CDDP resulted in an enhanced growth inhibitory effect on SW1736 and HTh-7 cells, suggesting that allicin may serve as an adjunctive 


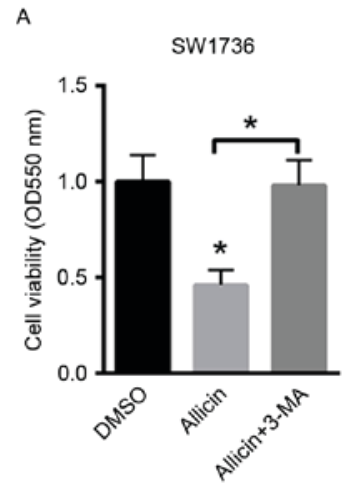

B

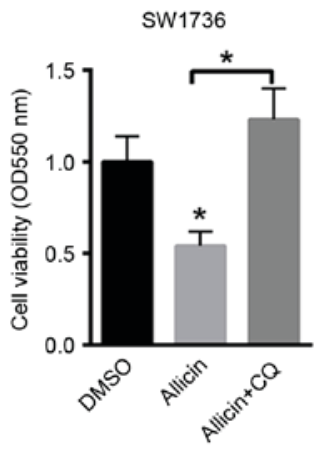

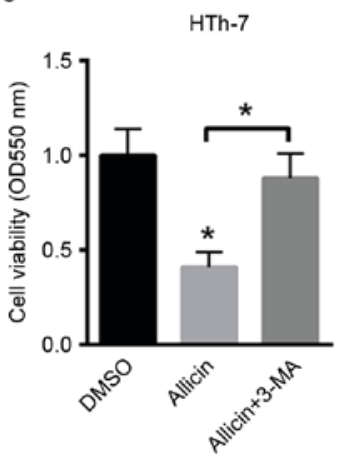

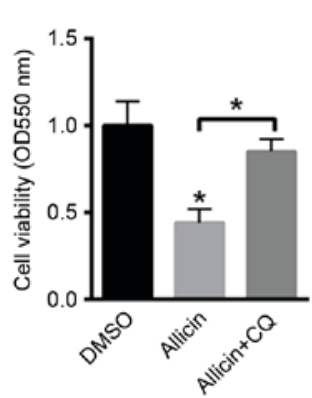

Figure 3. Allicin-induced SW1736 and HTh-7 cell death is associated with severe autophagy. Allicin-induced death of SW1736 cells was largely abolished by (A) 3-MA and (B) CQ. In HTh-7 cells, (C) 3-MA and (D) CQ inhibited allicin-induced cell death to a similar extent. "P<0.05 vs. control or as indicated. DMSO, dimethylsulfoxide; 3-MA, 3-methyladenine; $\mathrm{CQ}$, chloroquine; OD, optical density.

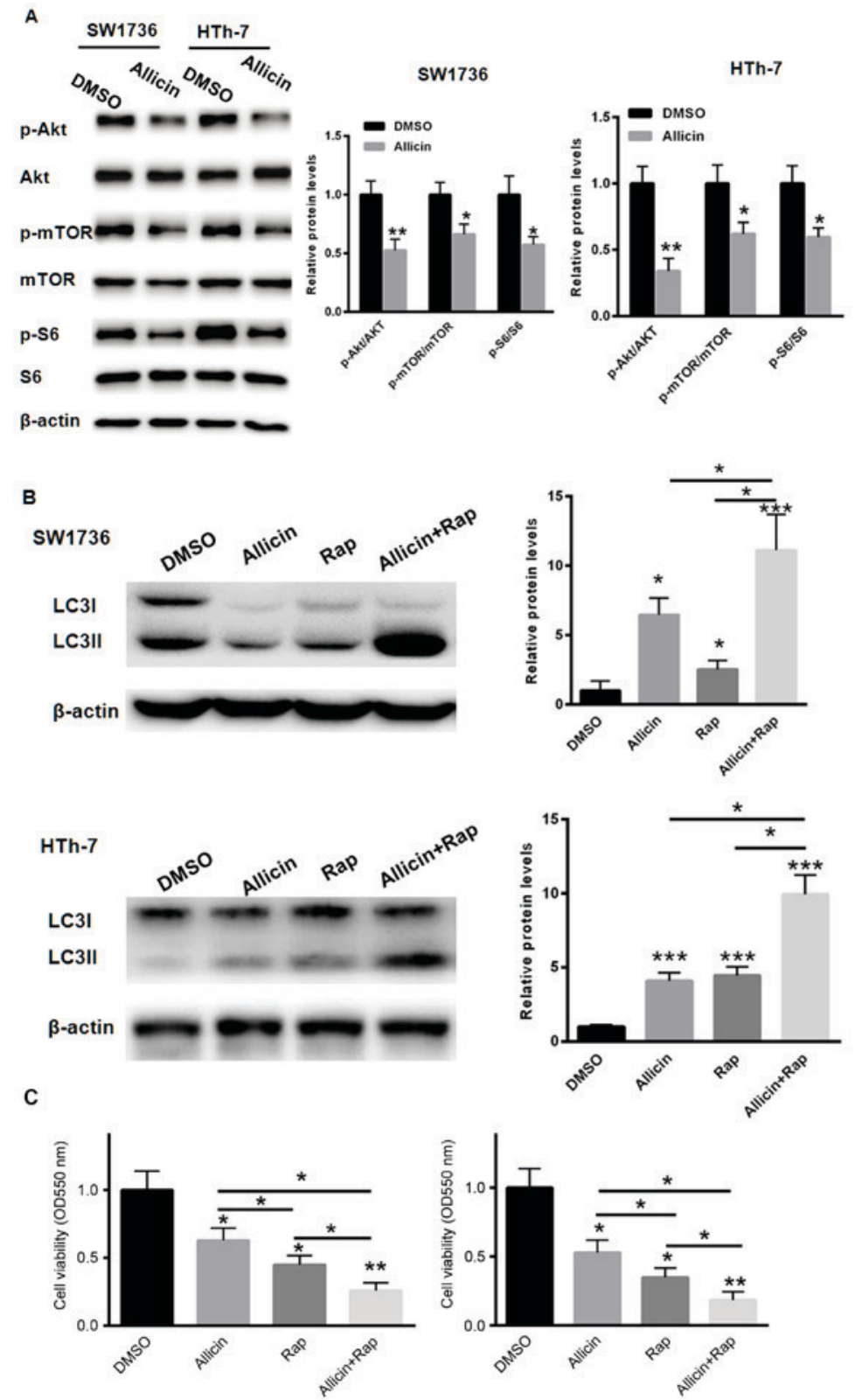

Figure 4. Allicin-induced SW1736 and HTh-7 cell autophagy is mainly achieved by suppression Akt/mTOR signaling. (A) Western blot analysis of associated proteins. (B) Combination treatment with allicin and rapamycin significantly enhanced the LC3II/LC3I ratio in SW1736 and HTh-7 cells compared with allicin or rapamycin alone. (C) Combined use of allicin and rapamycin induced more cell death compared with that of allicin or rapamycin alone. ${ }^{*} \mathrm{P}<0.05,{ }^{* * *} \mathrm{P}<0.01$, ${ }_{* * * *} \mathrm{P}<0.001$ vs. control or as indicated. p-mTOR, phosphorylated mammalian target of Rap; DMSO, dimethylsulfoxide; LC3, light chain 3; Rap, rapamycin; OD, optical density. 
therapy for thyroid cancer treatment. Previous studies have indicated that various drugs may enhance autophagy in thyroid cancer $(31,32)$. Vemurafenib has been revealed to induce endoplasmic reticulum stress response-mediated autophagy in thyroid cancer (31). The BH3 mimetic drug GX15-070 has been demonstrated to induce non-classical cell death with signs of apoptosis and autophagy in dedifferentiated thyroid carcinoma cells (32). The effect of allicin on SW1736 and HTh-7 cell autophagy was examined in the present study. The level of autophagy in SW1736 and HTh-7 cells was observed to be significantly increased in the presence of allicin. Of note, allicin-induced cell death was largely abolished by treatment with the autophagy inhibitors 3-MA or CQ, suggesting that allicin-induced SW1736 cell death was dependent on autophagy.

Since the Akt/mTOR signaling pathway has been reported to activate the process of autophagy $(33,34)$, the activation of Akt/mTOR signaling was evaluated after allicin treatment. Of note, allicin treatment decreased the activation of Akt, mTOR and S6. Furthermore, combined use of allicin and rapamycin induced more cell death compared with that induced by allicin or rapamycin alone. These results suggested that allicin triggered autophagy-dependent cell death through suppressing the Akt/mTOR signaling pathway.

In conclusion, the present study revealed that allicin may serve as an adjunctive therapy for thyroid cancer patients through inducing autophagy-dependent cell death even when apoptosis resistance is developed in cancer cells.

\section{References}

1. Sherman SI: Thyroid carcinoma. Lancet 361: 501-511, 2003.

2. Olejarski J, Yang M and Varghese J: Follicular thyroid carcinoma presenting as a soft tissue thigh mass. Lancet Diabetes Endocrinol 2: 348, 2014.

3. Spoto G, Esposito A, Santoleri F, Rubini C, Rutjes AW, Fioroni M, Ferrante $\mathrm{M}$ and Petrini $\mathrm{M}$ : Does cyclic guanosine monophosphate induce autophagy in thyroid malignant carcinoma through down-regulation of cyclic guanosine monophosphate phosphodiesterase? J Biol Regul Homeost Agents 30: 599-604, 2016.

4. Lu YC, Lee YR, Liao JD, Lin CY, Chen YY, Chen PT and Tseng YS: Reversine induced multinucleated cells, cell apoptosis and autophagy in human Non-small cell lung cancer cells. PLoS One 11: e0158587, 2016.

5. Saleh T, Cuttino L and Gewirtz DA: Autophagy is not uniformly cytoprotective: A personalized medicine approach for autophagy inhibition as a therapeutic strategy in Non-small cell lung cancer. Biochim Biophys Acta 1860: 2130-2136, 2016.

6. Chen MC, Chen CH, Liu YN, Wang HP, Pan SL and Teng CM: TW01001, a novel piperazinedione compound, induces mitotic arrest and autophagy in non-small cell lung cancer A549 cells. Cancer Lett 336: 370-378, 2013.

7. Choi JY, Hong WG, Cho JH, Kim EM, Kim J, Jung CH, Hwang SG, Um HD and Park JK: Podophyllotoxin acetate triggers anticancer effects against non-small cell lung cancer cells by promoting cell death via cell cycle arrest, ER stress and autophagy. Int J Oncol 47: 1257-1265, 2015.

8. Yang C, Ma X, Wang Z, Zeng X, Hu Z, Ye Z and Shen G: Curcumin induces apoptosis and protective autophagy in castration-resistant prostate cancer cells through iron chelation. Drug Des Devel Ther 11: 431-439, 2017.

9. Chen K and Shi W: Autophagy regulates resistance of non-small cell lung cancer cells to paclitaxel. Tumour Biol 37: 10539-10544, 2016.

10. Chen M, Du Y, Qui M, Wang M, Chen K, Huang Z, Jiang M, Xiong F, Chen J, Zhou J, et al: Ophiopogonin B-induced autophagy in non-small cell lung cancer cells via inhibition of the PI3K/Akt signaling pathway. Oncol Rep 29: 430-436, 2013.
11. Ma LN, Li LD, Li SC, Hao XM, Zhang JY, He P and Li YK: Allicin improves the cardiac function by protecting against apoptosis in rat model of myocardial infarction. Chin $\mathrm{J}$ Integr Med 23: 589-597, 2017.

12. Huang H, Jiang Y, Mao G, Yuan F, Zheng H, Ruan Y and Wu T: Protective effects of allicin on streptozotocin-induced diabetic nephropathy in rats. J Sci Food Agric 97: 1359-1366, 2017.

13. Ma T, Chen D, Tu Y, Zhang N, Si B, Deng K and Diao Q: Effect of supplementation of allicin on methanogenesis and ruminal microbial flora in Dorper crossbred ewes. J Anim Sci Biotechnol 7: 1, 2016.

14. Muller A, Eller J, Albrecht F, Prochnow P, Kuhlmann K, Bandow JE, Slusarenko AJ and Leichert LI: Allicin induces thiol stress in bacteria through S-Allylmercapto modification of protein cysteines. J Biol Chem 291: 11477-11490, 2016.

15. Sheppard JG and Long TE: Allicin-inspired thiolated fluoroquinolones as antibacterials against ESKAPE pathogens. Bioorg Med Chem Lett 26: 5545-5549, 2016.

16. Tu G, Zhang YF, Wei W, Li L, Zhang Y, Yang J and Xing Y: Allicin attenuates $\mathrm{H} 2 \mathrm{O} 2$-induced cytotoxicity in retinal pigmented epithelial cells by regulating the levels of reactive oxygen species. Mol Med Rep 13: 2320-2326, 2016.

17. Wang $S$ and Ren D: Allicin protects traumatic spinal cord injury through regulating the HSP70/Akt/iNOS pathway in mice. Mol Med Rep 14: 3086-3092, 2016.

18. Wang W, Du Z, Nimiya Y, Sukamtoh E, Kim D and Zhang G: Allicin inhibits lymphangiogenesis through suppressing activation of vascular endothelial growth factor (VEGF) receptor. J Nutr Biochem 29: 83-89, 2016.

19. Koleini N, Nickel BE, Wang J, Roveimiab Z, Fandrich RR, Kirshenbaum LA, Cattini PA and Kardami E: Fibroblast growth factor-2-mediated protection of cardiomyocytes from the toxic effects of doxorubicin requires the mTOR/Nrf-2/HO-1 pathway. Oncotarget 8: 87415-87430, 2017.

20. Giuffrida R, Adamo L, Iannolo G, Vicari L, Giuffrida D, Eramo A, Gulisano M, Memeo L and Conticello C: Resistance of papillary thyroid cancer stem cells to chemotherapy. Oncol Lett 12: 687-691, 2016.

21. de Groot S, Janssen LG, Charehbili A, Dijkgraaf EM, Smit VT, Kessels LW, van Bochove A, van Laarhoven HW, Meershoek-Klein Kranenbarg E, van Leeuwen-Stok AE, et al: Thyroid function alters during neoadjuvant chemotherapy in breast cancer patients: Results from the NEOZOTAC trial (BOOG 2010-01). Breast Cancer Res Treat 149: 461-466, 2015.

22. Denaro N, Nigro CL, Russi EG and Merlano MC: The role of chemotherapy and latest emerging target therapies in anaplastic thyroid cancer. Onco Targets Ther 9: 1231-1241, 2013.

23. De Besi P, Busnardo B, Toso S, Girelli ME, Nacamulli D, Simioni N, Casara D, Zorat P and Fiorentino MV: Combined chemotherapy with bleomycin, adriamycin, and platinum in advanced thyroid cancer. J Endocrinol Invest 14: 475-480, 1991.

24. Droz JP, Rougier P, Goddefroy V, Schlumberger M, Gardet P and Parmentier C: Chemotherapy for medullary cancer of the thyroid. Phase II trials with adriamycin and cis-platinum administered as monochemotherapy. Bull Cancer 71: 195-199, 1984.

25. Tirro E, Consoli ML, Massimino M, Manzella L, Frasca F, Sciacca L, Vicari L, Stassi G, Messina L, Messina A and Vigneri P: Altered expression of c-IAP1, survivin, and Smac contributes to chemotherapy resistance in thyroid cancer cells. Cancer Res 66: 4263-4272, 2006.

26. Albero A, Lopéz JE, Torres A, de la Cruz L and Martín T: Effectiveness of chemotherapy in advanced differentiated thyroid cancer: A systematic review. Endocr Relat Cancer 23: R71-R84, 2016.

27. Lacin S, Esin E, Karakas Y and Yalcin S: Metastatic medullary thyroid cancer: A dramatic response to a systemic chemotherapy (temozolomide and capecitabine) regimen. Onco Targets Ther 8: 1039-1042, 2015.

28. Ali AB, Nin DS, Tam J and Khan M: Role of chaperone mediated autophagy (CMA) in the degradation of misfolded N-CoR protein in non-small cell lung cancer (NSCLC) cells. PLoS One 6: e25268, 2011.

29. Giatromanolaki A, Kalamida D, Sivridis E, Karagounis IV, Gatter KC, Harris AL and Koukourakis MI: Increased expression of transcription factor EB (TFEB) is associated with autophagy, migratory phenotype and poor prognosis in non-small cell lung cancer. Lung Cancer 90: 98-105, 2015.

30. Guo Q, Liu Z, Jiang L, Hu T, Li D, Liu Y, Liu M, Liang X and Nan K: Starvation-induced autophagy in cultured non-small cell lung cancer cells. Nan Fang Yi Ke Da Xue Xue Bao 34: 627-630, 2014. 
31. Wang W, Kang H, Zhao Y, Min I, Wyrwas B, Moore M, Teng L, Zarnegar R, Jiang $X$ and Fahey TJ III: Targeting autophagy sensitizes BRAF-Mutant thyroid cancer to vemurafenib. J Clin Endocrinol Metab 102: 634-643, 2017.

32. Broecker-Preuss M, Viehof J, Jastrow H, Becher-Boveleth $\mathrm{N}$, Fuhrer D and Mann K: Cell death induction by the BH3 mimetic GX15-070 in thyroid carcinoma cells. J Exp Clin Cancer Res 34: 69, 2015.
33. Hwang KE, Kim YS, Jung JW, Kwon SJ, Park DS, Cha BK, Oh SH, Yoon KH, Jeong ET and Kim HR: Inhibition of autophagy potentiates pemetrexed and simvastatin-induced apoptotic cell death in malignant mesothelioma and non-small cell lung cancer cells. Oncotarget 6: 29482-29496, 2015.

34. Jeong EH, Choi HS,Lee TG, Kim HR and Kim CH: Dual inhibition of PI3K/Akt/mTOR pathway and role of autophagy in Non-small cell lung cancer cells. Tuberc Respir Dis (Seoul) 72: 343-351, 2012. 